\title{
Morfologia polínica de espécies do gênero Merremia Dennst. (Convolvulaceae) ocorrentes no Estado da Bahia, Brasil ${ }^{1}$
}

\author{
Kelly Regina Batista Leite ${ }^{2,4}$, Rosângela Simão-Bianchini ${ }^{3}$ e Francisco de Assis Ribeiro dos Santos ${ }^{2}$
}

Recebido em 03/03/2004. Aceito em 10/09/2004

\begin{abstract}
RESUMO - (Morfologia polínica de espécies do gênero Merremia Dennst. (Convolvulaceae) ocorrentes no Estado da Bahia, Brasil). O gênero Merremia (Convolvulaceae) teve as espécies nativas na Bahia inventariadas e estudadas sob o ponto de vista palinológico. Foram registrados nove táxons do gênero: M. aegyptia (L.) Urb., M. cissoides (Lam.) Hallier f., M. digitata (Spreng.) Hall. f. var. digitata, M. digitata var. ericoides (Meissn.) Austin \& Staples, M. dissecta (Jacq.) Hall. f. var. edentata (Meisn.) O’Donell, M. flagellaris (Choisy) O'Donell, M. macrocalyx (Ruiz et Pav.) O'Donell, M. tomentosa (Choisy) Hall. f. e M. umbellata (L.) Hall. f. Em M. digitata var. digitata e $M$. flagellaris os grãos de pólen apresentavam-se irregularmente e com três e seis colpos; membrana apertural espessa em $M$. aegyptia, M. cissoides, $M$. digitata var. ericoides; a exina é espessa e granulada em todas as espécies, o teto é fino; sexina mais espessa que nexina. Os grãos de pólen com 6 aberturas são zonocolpados (M. umbellata) ou pantocolpados ( . digitata var. digitata, M. digitata var. ericoides, e $M$. flagellaris), podendo ocorrer em um mesmo espécime mais de um tipo apertural de grão de pólen. Os dados palinológicos observados vêm fortalecer a união das espécies $M$. aegyptia, M. dissecta var. edentata e M. macrocalyx em M. sect. Schizips, M. umbellata em $M$. sect. Xanthips e as demais em $M$. sect. Cissoides. Nesta última seção, com exceção de $M$. tomentosa, as espécies restantes pertencem ao complexo $M$. digitata. No referido complexo as espécies não estão bem delimitadas sendo necessário que estudos mais profundos sejam realizados afim de melhor delimitá-las.
\end{abstract}

Palavras-chave: Convolvulaceae, Merremia, Palinologia, Bahia, Brasil

\begin{abstract}
Morphology pollen of species genus Merremia Dennst. (Convolvulaceae) ocurring in Bahia State, Brazil). Species of the genus Merremia (Convolvulaceae) from State of Bahia were surveyed, and their pollen grains were studied by usual methods. Nine taxa were recorded: M. aegyptia (L.) Urb., M. cissoides (Lam.) Hallier f., M. digitata (Spreng.) Hall. f. var. digitata, M. digitata var. ericoides (Meissn.) Austin \& Staples, M. dissecta (Jacq.) Hall. f. var. edentata (Meisn.) O'Donell, M. flagellaris (Choisy) O’Donell, M. macrocalyx (Ruiz et Pav.) O'Donell, M. tomentosa (Choisy) Hall. f. and M. umbellata (L.) Hall. f. In M. digitata var. digitata and M. flagellaris, the pollen grains presented irregular colpi and with three and six long colpi. Thick apertural membrane was observed in M. aegyptia, $M$. cissoides, $M$. digitata var. ericoides; presence with opercules in M. aegyptia, $M$. cissoides and $M$. digitata var. ericoides pollen grains. Six-aperturated pollen grains are zonocolpate (in $M$. umbellata) or pantocolpate (in M. digitata var. digitata, M. digitata var. ericoides and $M$. flagellaris); more than one apertural type can be found grains in the same specimen. The palinological data corroborate the placement of M. aegyptia, M. dissecta var. edentata and M. macrocalyx in M. sect. Schizips, M. umbellata in M. sect. Xanthips and other ones in $M$. sect. Cissoides. In the latter section, excluding $M$. tomentosa, all species belong to the complex M. digitata, in which they are not well delimitated.
\end{abstract}

Key words: Convolvulaceae, Merremia, Palinology, Bahia, Brazil

\section{Introdução}

A família Convolvulaceae tem distribuição, principalmente, tropical com representantes em climas subtropicais e temperados (Barroso et al. 1986; Mabberley 1987; Ribeiro \& Bianchini 1999). No Brasil, Meissner (1869) reconheceu cerca de 312 espécies que ocorrem nas mais diversas formações vegetais.
O gênero Merremia Dennst., de acordo com Austin (1979), tem distribuição tropical e subtropical com mais de 60 espécies e pelo menos 30 delas são encontradas no Novo Mundo (Austin \& Staples 1983). Um dos trabalhos taxonômicos mais completos do gênero é a revisão feita por O’Donell (1941) para as espécies americanas, tratando 28 espécies. Nesse trabalho o autor distribuiu as espécies de Merremia em cinco

\footnotetext{
Parte da Dissertação de Mestrado da primeira Autora

2 Universidade Estadual de Feira de Santana, Departamento de Ciências Biológicas, Km 3, BR 116 Norte, Campus Universitário, CEP 44031-460, Feira de Santana, Bahia, Brasil (fasantos@uefs.br)

3 Instituto de Botânica da Secretaria de Meio Ambiente do Estado de São Paulo, C. Postal 4005, CEP 01061-970, São Paulo, São Paulo, Brasil

4 Autor para correspondência: kellyleite03@hotmail.com
} 
seções, criadas com base em características macromorfológicas.

No Brasil, o gênero Merremia, possui 15 espécies, de acordo com levantamentos realizados em herbários e literatura. No Estado da Bahia foram encontradas os seguintes táxons: M. aegyptia (L.) Urban, M. cissoides (Lam.) Hall. f., M. digitata (Spreng.) Hall. f. var. digitata, $M$. digitata var. ericoides (Meissn.) Austin \& Staples, M. dissecta (Jacq.) Hall. f. var. edentata (Meisn.) O’Donell, M. flagellaris (Choisy) O’Donell, M. macrocalyx (Ruiz et Pav.) O'Donell, M. tomentosa (Choisy) Hall. f. e M. umbellata (L.) Hall. f.

O primeiro trabalho palinotaxonômico sobre as Convolvulaceae foi o de Hallier (1893), no qual a família foi dividida em dois grandes grupos (Echinoconieae e Psiloconiae) baseado no padrão apertural da exina e do tipo apertural do grão de pólen. Posteriormente, vários autores trataram do pólen da família Convolvulaceae, tais como Erdtman (1952), Laguardia (1961), Lewis \& Oliver (1965), Sengupta (1972), Ferguson et al. (1977), Melhem \& Corrêa (1987) e Araújo et al. (2000).

Os estudos palinológicos mais recentes com espécies de Convolvulaceae são os desenvolvidos por Araújo et al. (2000), que estudaram os grãos de pólen de 10 gêneros e 24 espécies de Convolvulaceae que ocorrem na Bahia, entre elas três espécies de Merremia. Pozhidaev (2000) estudou a variedade e o padrão apertural em grãos de pólen de famílias de Monocotiledôneas e Dicotiledôneas, sendo neste último trabalho incluída M. pterygocaulos (Stend.) Hall. f. Carreira \& Barth (2003) estudaram a flora polínica da vegetação de "cangas" da Serra de Carajás, na qual está presente $M$. macrocalyx.

$\mathrm{O}$ presente estudo teve como objetivo a caracterização da morfologia polínica das espécies do gênero Merremia do Estado da Bahia, visando contribuir para melhor delimitação taxonômica das mesmas.

\section{Material e métodos}

O material polinífero analisado foi retirado de exsicatas depositadas nos herbários ALCB, CEPEC, HRB, HUEFS, IPA, SPF e UB (siglas conforme Holmgren et al. 1990). A preparação dos grãos de pólen seguiu o método padrão da acetólise (Erdtman 1960).

Os diâmetros (polar e equatorial) foram medidos aleatoriamente em 25 grãos de pólen (sempre que possível). Para medir a espessura da exina (sexina e nexina) foram realizadas as medidas em dez grãos de pólen. Foram feitas observações em microscopia eletrônica de varredura (MEV), sem que os grãos de pólen fossem submetidos ao tratamento acetolítico. As descrições palinológicas foram feitas tomando-se como base a nomenclatura de Punt et al. (1994). As lâminas preparadas encontram-se depositadas na Palinoteca do Laboratório de Micromorfologia Vegetal da Universidade Estadual de Feira de Santana.

\section{Resultados e discussão}

Descrição palinológica dos táxons de Merremia ocorrentes na Bahia - As medidas dos diâmetros e das camadas da exina das espécies estudadas encontram-se nas Tab. 1 e 2, respectivamente.

\section{Merremia aegyptia (L.) Urban}

Fig. 1-3.

Grãos de pólen em mônades, grandes, isopolares, oblato-esferoidais a subprolatos, amb (sub)circular, 3-colpados, granulados. Colpos longos de contorno irregular e extremidades afiladas com membrana apertural espessa (Fig. 1). Exina espessa, com grânulos heterogêneos em tamanho e irregularmente distribuídos, só visíveis em MEV; teto fino e ondulado em corte óptico; columelas delgadas, longas, irregularmente distribuídas, às vezes fundidas em grupos de 2-3 unidades; sexina mais espessa que a nexina.

Merremia cissoides (Lam.) Hall. f.

Fig. 4-5.

Grãos de pólen em mônades, grandes, isopolares, subprolatos, amb circular, 3(-4)-colpados ou 6-pantocolpados, granulados. Colpos longos com contorno irregular e extremidades afiladas, com membrana apertural espessa. Exina espessa, granulada, com teto fino e ondulado em corte óptico; columelas delgadas, longas, heterogêneas, regularmente distribuídas; sexina mais espessa que a nexina. Sob MEV, o teto apresenta-se com grânulos diminutos e densamente distribuídos (Fig. 5).

\section{Merremia digitata (Sprengel) Hall. f. var. digitata}

Fig. 6-12.

Grãos de pólen em mônades, grandes, isopolares, subprolatos, amb (sub)circular, 3-colpados (Fig. 6-7) e 6-pantocolpados (Fig. 11-12), raramente sincolpados (Fig. 8-10), granulados. Colpos longos com contorno regular e extremidades afiladas. Exina espessa, com teto fino e ondulado em corte óptico, columelas longas, 
delgadas, regularmente distribuídas, algumas possuindo bifurcações na extremidade distal; sexina mais espessa que a nexina.

Merremia digitata var. ericoides (Meisn.) Austin \& Staples

Fig. 13-16.

Grãos de pólen em mônades, grandes, isopolares, prolato-esferoidais, amb (sub)circular, 3-colpados e 6-pantocolpados, granulados. Colpos longos com contorno irregular e extremidades afiladas; com membrana apertural espessa (Fig. 15). Exina espessa, teto fino, ondulado em corte óptico; columelas delgadas, curtas e regularmente distribuídas; sexina mais espessa que a nexina. Diminutos grânulos são visíveis sob MEV (Fig. 14-16) e também sob microscopia óptica (MO).

Merremia dissecta (Jacq.) Hall. f. var. edentata Fig. 17-21.

Grãos de pólen em mônades, grandes a muito grandes, isopolares, prolato-esferoidais a subprolatos, amb (sub)circular, 3-colpados. Colpos longos com contorno regular e extremidades afiladas, membrana apertural fortemente granulada (Fig. 19-21). Exina espessa, levemente mais espessa nas proximidades dos colpos do que no mesocolpo; columelas delgadas, curtas; sexina mais espessa que a nexina; com grânulos

Tabela 1. Caracteres morfométricos dos grãos de pólen das espécies de Merremia Dennst. (Convolvulaceae) ocorrentes na Bahia, Brasil. FV- Faixa de variação; * - medida em Dep; Irr. - irregularmente colpado; x média.

\begin{tabular}{|c|c|c|c|c|c|}
\hline Espécie/Espécime/Herbário & $\begin{array}{c}\text { Diâmetro polar }(\mu \mathrm{m}) \\
\text { FV(x)FV }\end{array}$ & $\begin{array}{c}\text { Diâmetro equatorial }(\mu \mathrm{m}) \\
\qquad \mathrm{FV}(\mathrm{x}) \mathrm{FV}\end{array}$ & $\mathrm{P} / \mathrm{E}$ & $\begin{array}{c}\text { Espessura } \\
\text { da exina }(\mu \mathrm{m})\end{array}$ & Aberturas \\
\hline \multicolumn{6}{|l|}{ Merremia aegyptia } \\
\hline K.R.B. Leite et al. 142 (HUEFS) & $67,50(74,00) 87,50$ & $52,50(60,70) 72,50$ & 1,21 & 4,20 & 3 \\
\hline L.R. Noblick 4139 (HUEFS) & $55,00(73,20) 97,50$ & $65,00(74,70) 80,00$ & 0,97 & 4,50 & 3 \\
\hline M.J.S. Lemos et al. 146 (HUEFS) & $52,50(67,50) 72,50$ & $45,00(55,20) 60,00$ & 1,22 & 4,12 & 3 \\
\hline \multicolumn{6}{|l|}{ M. cissoides } \\
\hline R.M. Harley et al. 53440 (HUEFS) & - & $47,50(59,30) 67,50 *$ & - & 4,32 & 4,6 \\
\hline L.P. de Queiroz 1300 (HUEFS) & $52,50(58,24) 67,50$ & $40,00(44,60) 52,50$ & 1,30 & 4,80 & 3 \\
\hline L.B. da Silva et al. 96 (HRB) & $50,00(60,00) 67,50$ & $40,00(45,80) 50,00$ & 1,31 & 3,45 & 3 \\
\hline \multicolumn{6}{|l|}{ M. digitata var. digitata } \\
\hline N. Hind 4393 (HUEFS) & $75,00(81,70) 100,00$ & $55,00(77,70) 80,00$ & 1,32 & 4,90 & $3(6)$ \\
\hline M.A. Silva et al. 1355 (UB) & $80,00(84,70) 90,00$ & $55,00(60,30) 65,00$ & 1,40 & 4,42 & $(\operatorname{Irr}) 3(6)$ \\
\hline R.M. Harley 21087 (CEPEC) & $75,00(80,50) 87,50$ & $47,50(60,90) 70,00$ & 1,32 & 4,65 & $3(6)$ \\
\hline \multicolumn{6}{|l|}{ M. digitata var. ericoides } \\
\hline L.R. Noblick 3806 (HUEFS) & - & $52,50(61,60) 75,00 *$ & - & 5,25 & 3 \\
\hline H.P. Bautista 1254 (HRB) & $55,00(69,10) 80,00$ & $42,50(60,80) 70,00$ & 1,13 & 5,60 & $3(6)$ \\
\hline \multicolumn{6}{|l|}{ M. dissecta var. edentata } \\
\hline K.R.B. Leite 141 (HUEFS) & $67,50(84,70) 97,50$ & $75,00(76,40) 107,50$ & 1,10 & 4,10 & 3 \\
\hline R.M. Harley 17232 (IPA) & - & $87,50(103,30) 120,00 *$ & - & 4,42 & 3 \\
\hline M.M. Santos et al. 121 (CEPEC) & $77,50(83,20) 87,50$ & $57,50(63,00) 67,50$ & 1,32 & 3,45 & 3 \\
\hline \multicolumn{6}{|l|}{ M. flagellaris } \\
\hline N. Hind 4183 (CEPEC) & $67,50(76,80) 92,50$ & $45,00(62,70) 77,50$ & 1,22 & 4,20 & 3 \\
\hline M.L. Guedes 5512 (SPF) & $75,00(94,42) 95,00$ & $62,50(67,20) 75,00$ & 1,40 & 5,25 & $3(6)$ \\
\hline R.M. Harley s.n. PCD 3664 (ALCB) & $69,50(91,56) 98,50$ & $55,50(65,50) 76,00$ & 1,39 & 5,25 & (Irr)3 \\
\hline \multicolumn{6}{|l|}{ M. macrocalyx } \\
\hline K.R.B. Leite 49 (HUEFS) & $75,00(85,60) 97,50$ & $52,50(63,30) 75,00$ & 1,35 & 4,60 & 3 \\
\hline R.M. Harley 20010 (IPA) & $77,50(94,62) 105,00$ & $65,00(74,25) 82,50$ & 1,27 & 4,27 & 3 \\
\hline L. Corandin et al. $6052(\mathrm{CEN})$ & $76,80(88,76) 98,50$ & $66,50(70,45) 85,00$ & 1,25 & 4,86 & 3 \\
\hline \multicolumn{6}{|l|}{ M. tomentosa } \\
\hline R.M. Harley 21202 (CEPEC) & $55,00(64,00) 77,50$ & $37,50(49,10) 62,50$ & 1,30 & 3,90 & 3 \\
\hline E.F. Almeida 253 (HUEFS) & $57,50(66,05) 70,00$ & $42,50(47,67) 52,50$ & 1,38 & 4,12 & 3 \\
\hline \multicolumn{6}{|l|}{ M. umbellata } \\
\hline G.C.P. Pinto 84/86 (IPA) & - & $75,00(81,10) 87,50 *$ & - & 6,07 & 6 \\
\hline R.S. Pinheiro 1234 (CEPEC) & $70,00(77,12) 87,50$ & $52,50(66,22) 72,50$ & 1,17 & 6,15 & 6 \\
\hline F. França 2335 (HUEFS) & - & $57,50(70,00) 82,50 *$ & - & 6,00 & 6 \\
\hline
\end{tabular}


visíveis sob MEV (Fig. 21) e também sob microscopia óptica (MO).

\section{Merremia flagellaris (Choisy) O’Donell}

Fig. 22-25.

Grãos de pólen em mônades, grandes, isopolares, subprolatos, amb (sub)circular, 3-colpados e 6-pantocopaldos (raramente sincolpados). Colpos longos com contorno regular e extremidades afiladas; com membrana apertural bem visível. Exina espessa, teto fino, liso em corte óptico; columelas delgadas, longas, algumas vezes fundidas em grupos de 2-3 e regularmente distribuídas; sexina mais espessa que a nexina.

\section{Merremia macrocalyx (Ruiz et Pav.) O'Donell}

Fig. 26-30.
Grãos de pólen em mônades, grandes, isopolares, prolatos, amb circular, 3-colpados. Colpos longos com extremidades afiladas, membrana apertural granulada. Exina espessa, teto fino e liso em corte óptico; columelas delgadas, longas e ramificadas distalmente; sexina mais espessa que a nexina.

Merremia tomentosa (Choisy) Hall. f.

Fig. 31-33.

Grãos de pólen em mônades, grandes, isopolares, subprolatos, amb (sub)circular, 3-colpados. Colpos longos com contorno irregular e extremidades afiladas; membrana apertural com granulação tênue. Exina espessa, teto fino, columelas delgadas; sexina mais espessa que a nexina.

Tabela 2. Espessura da exina dos grãos de pólen das espécies de Merremia Dennst. (Convolvulaceae) ocorrentes na Bahia, Brasil.

\begin{tabular}{|c|c|c|c|}
\hline Espécie/Espécime/Herbário & $\begin{array}{l}\text { Espessura da exina } \\
x(\mu \mathrm{m})\end{array}$ & $\begin{array}{l}\text { Espessura da sexina } \\
\qquad \mathrm{x}(\mu \mathrm{m})\end{array}$ & $\begin{array}{l}\text { Espessura da nexina } \\
\mathrm{x}(\mu \mathrm{m})\end{array}$ \\
\hline \multicolumn{4}{|l|}{ Merremia aegyptia } \\
\hline K.R.B. Leite et al. 142 (HUEFS) & 4,20 & 3,07 & 1,13 \\
\hline L.R. Noblick 4139 (HUEFS) & 4,50 & 2,90 & 1,60 \\
\hline M.J.S. Lemos et al. 146 (HUEFS) & 4,12 & 2,77 & 1,35 \\
\hline \multicolumn{4}{|l|}{ M. cissoides } \\
\hline R.M. Harley et al. 53440 (HUEFS) & 4,32 & 3,00 & 1,32 \\
\hline L.P. de Queiroz 1300 (HUEFS) & 4,80 & 3,30 & 1,50 \\
\hline L.B. da Silva et al. 96 (HRB) & 3,45 & 2,32 & 1,12 \\
\hline \multicolumn{4}{|l|}{ M. digitata var. digitata } \\
\hline N. Hind 4393 (HUEFS) & 4,90 & 3,60 & 1,30 \\
\hline M.A. Silva et al. 1355 (UB) & 4,42 & 3,22 & 1,20 \\
\hline R.M. Harley 21087 (CEPEC) & 4,65 & 3,30 & 1,35 \\
\hline \multicolumn{4}{|l|}{ M. digitata var. ericoides } \\
\hline L.R. Noblick 3806 (HUEFS) & 5,25 & 2,70 & 2,55 \\
\hline H.P. Bautista 1254 (HRB) & 5,60 & 4,20 & 1,40 \\
\hline \multicolumn{4}{|l|}{ M. dissecta var. edentata } \\
\hline K.R.B. Leite 141 (HUEFS) & 4,10 & 2,60 & 1,50 \\
\hline R.M. Harley 17232 (IPA) & 4,42 & 3,15 & 1,27 \\
\hline M.M. Santos et al. 121 (CEPEC) & 3,45 & 2,47 & 0,98 \\
\hline \multicolumn{4}{|l|}{ M. flagellaris } \\
\hline N. Hind 4183 (CEPEC) & 4,20 & 3,00 & 1,20 \\
\hline M.L. Guedes 5512 (SPF) & 5,25 & 3,82 & 1,43 \\
\hline R.M. Harley s.n. PCD 3664 (ALCB) & 5,25 & 3,76 & 1,49 \\
\hline \multicolumn{4}{|l|}{ M. macrocalyx } \\
\hline K.R.B. Leite 49 (HUEFS) & 4,60 & 3,52 & 1,08 \\
\hline R.M. Harley 20010 (IPA) & 4,27 & 2,92 & 1,35 \\
\hline L. Corandin et al. 6052 (CEN) & 4,86 & & \\
\hline \multicolumn{4}{|l|}{ M. tomentosa } \\
\hline R.M. Harley 21202 (CEPEC) & 3,90 & 2,55 & 1,35 \\
\hline E.F. Almeida 253 (HUEFS) & 4,12 & 2,75 & 1,80 \\
\hline \multicolumn{4}{|l|}{ M. umbellata } \\
\hline G.C.P. Pinto 84/86 (IPA) & 6,07 & 4,57 & 1,50 \\
\hline R.S. Pinheiro 1234 (CEPEC) & 6,15 & 4,65 & 1,50 \\
\hline F. França 2335 (HUEFS) & 6,00 & 4,50 & 1,50 \\
\hline
\end{tabular}


Merremia umbellata (L.) Hall. f.

Fig. 34-36.

Grãos de pólen em mônades, grandes, subprolatos, amb circular, 6-zonocolpados. Colpos longos com contorno irregular e extremidades afiladas, membrana apertural granulada. Exina espessa, teto fino em corte óptico, columelas delgadas, longas; sexina mais espessa que a nexina.

Na maioria das preparações, os grãos de pólen apresentaram-se muito amassados longitudinalmente (Fig. 22, 26, 33, 34), evidenciando assim a flexibilidade de sua exina. Isto também é facilitado pelas aberturas do tipo colpado. Consequientemente, a forma do grão de pólen mostrou-se tendendo à condição prolata (P/E > 1), exceção feita apenas ao material examinado de $M$. aegyptia (Noblick 4139-HUEFS) que apresentou grãos de pólen tendendo à forma oblatoesferoidal.

No que se refere aos valores médios dos diâmetros, os grãos de pólen das espécies ficaram na classe de tamanho grande, cujos valores extremos (menor e maior) foram observados em $M$. cissoides $(44,60 \mu \mathrm{m})$ e $M$. dissecta var. edentata $(103,30 \mu \mathrm{m})$, respectivamente.

Entre as espécies estudadas, M. aegyptia destacou-se por apresentar a maior faixa de variação no que se refere ao tamanho, pois no espécime $L . R$. Noblick 4139 (HUEFS) desta espécie, o diâmetro polar teve amplitude de $42,50 \mu \mathrm{m}$.

M. dissecta var. edentata (R.M. Harley 17232) em DP e M. flagellaris ( $N$. Hind 4183) em DE foram as outras espécies que também apresentaram grande amplitude $(32,5 \mu \mathrm{m})$ na faixa de variação do tamanho dos grãos de pólen; sendo possível atribuir este fato a sua baixa resistência ao processo de acetólise, uma vez que com a destruição da membrana apertural, os colpos se abrem mais, aumentando, assim, as medidas do diâmetro equatorial.

A maioria das espécies apresentou grãos de pólen com três aberturas, entretanto, algumas tinham grãos de pólen com número de aberturas diferentes deste número básico, demonstrando assim a existência ou não de heteromorfismo polínico nas espécies. Isto também foi observado por Pozhidaev (2000) em M. pterygocaulos (Stend.) Hall. f., cujos grãos de pólen são 3-4-colpados.

Wodehouse (1935), Erdtman (1952) e Dajoz et al. (1995), em seus trabalhos, mostraram que a variação no nível de ploidia do gametófito é normalmente considerada como a causa do heteromorfismo polínico, quanto ao número de aberturas. Neste estudo, esse nível de variação pode ser observado em $M$. cissoides, pois dois espécimes analisados apresentaram grãos de pólen 3-colpados, e um espécime (R.M. Harley et al. 53440-HUEFS) grãos de pólen 4-6 zonocolpados. O inverso pode ser referido para M. flagellaris pois dois dos espécimes analisados eram heteromórficos para o número de aberturas e outro ( $N$. Hind 4183-CEPEC) monomófico, com grãos de pólen 3-colpados.

Nos espécimes M.A. Silva et al. 1355 (UB) de M. digitata var. digitata e R.M. Harley s.n. PCD 3664 (ALCB) de M. flagellaris foram observados grãos de pólen do tipo irregularmente colpado (Fig. 8). As aberturas são fusionadas sinuosamente como a costura numa bola de tênis. Grãos de pólen com esta mesma conformação apertural foram referidos para táxons de várias outras famílias. Leuenberger (1976), por exemplo, indicou tais grãos de pólen para espécies de Cactaceae. Segundo Pozhidaev (2000), tal tipo polínico seria a forma intermediária (anterior) na evolução morfopolínica cujo final seria o tipo 4-colpado. Este autor usa, inclusive, M. pterygocaulos para exemplificar uma seqüência evolutiva na qual grãos de pólen irregularmente colpados estão presentes.

Pozhidaev (2000) cita, ainda, para M. pterygocaulos grãos de pólen 4-colpados nos quais um dos pares de colpos fusiona-se num dos pólos. Este tipo polínico também foi observado em $M$. digitata var. digitata (Fig. 8-9).

Considerando a exina, é preciso ressaltar a baixa espessura (Tab. 2) e transparência do teto que muitas vezes dificultam a sua visualização; são também os diminutos elementos esculturais - grânulos (Fig. 5, 16 e 21) só observáveis sob MEV. Os grânulos por serem de reduzido tamanho são de difícil fotografia mesmo no aparelho de MEV, utilizado pela necessidade de grandes magnitudes de aumento.

Implicações taxonômicas da morfologia polínica - De acordo com as observações feitas por Hallier (1893), as espécies de Merremia analisadas pertencem ao grupo "Psiloconiae", por apresentarem grãos de pólen com ornamentação da exina perfurada (não espinhosa). Alguns autores, no entanto, como Laguardia (1961) e Palácios-Chávez et al. (1991) mencionaram a exina granulada ou verrucada para espécies do gênero Merremia. Como estes autores, neste trabalho, tais tipos de ornamentação da exina foram observados nos espécimes analisados (excetuando o tipo verrucado). Devido ao fato de as columelas serem vistas através do teto "fino" como algo supra-tectal, característica também observada por Melhem \& Corrêa (1987) 
analisando $M$. macrocalyx, alguns autores indicam a presença de elementos supratectais nos grãos de pólen das espécies de Merremia. Mais recentemente, essa espécie teve seus grãos de pólen descritos como psilados por Carreira \& Barth (2003).

$M$. aegyptia, $M$. dissecta var. edentata e $M$. macrocalyx apresentaram em todos os materiais examinados apenas grãos de pólen do tipo 3-colpado. Isto corrobora a proposta de O'Donell (1941) que as reuniu numa mesma seção com base em caracteres macromorfológicos (Merremia sect. Cissoides).

Laguardia (1961), no entanto, observou que M. dissecta e M. macrocalyx apresentavam grãos de pólen 4-colpados em espécimes uruguaios. Divergindo assim dos resultados aqui apresentados para estas espécies.

M. umbellata foi a única espécie que apresentou grãos de pólen do tipo 6-zonocolpado, o que também
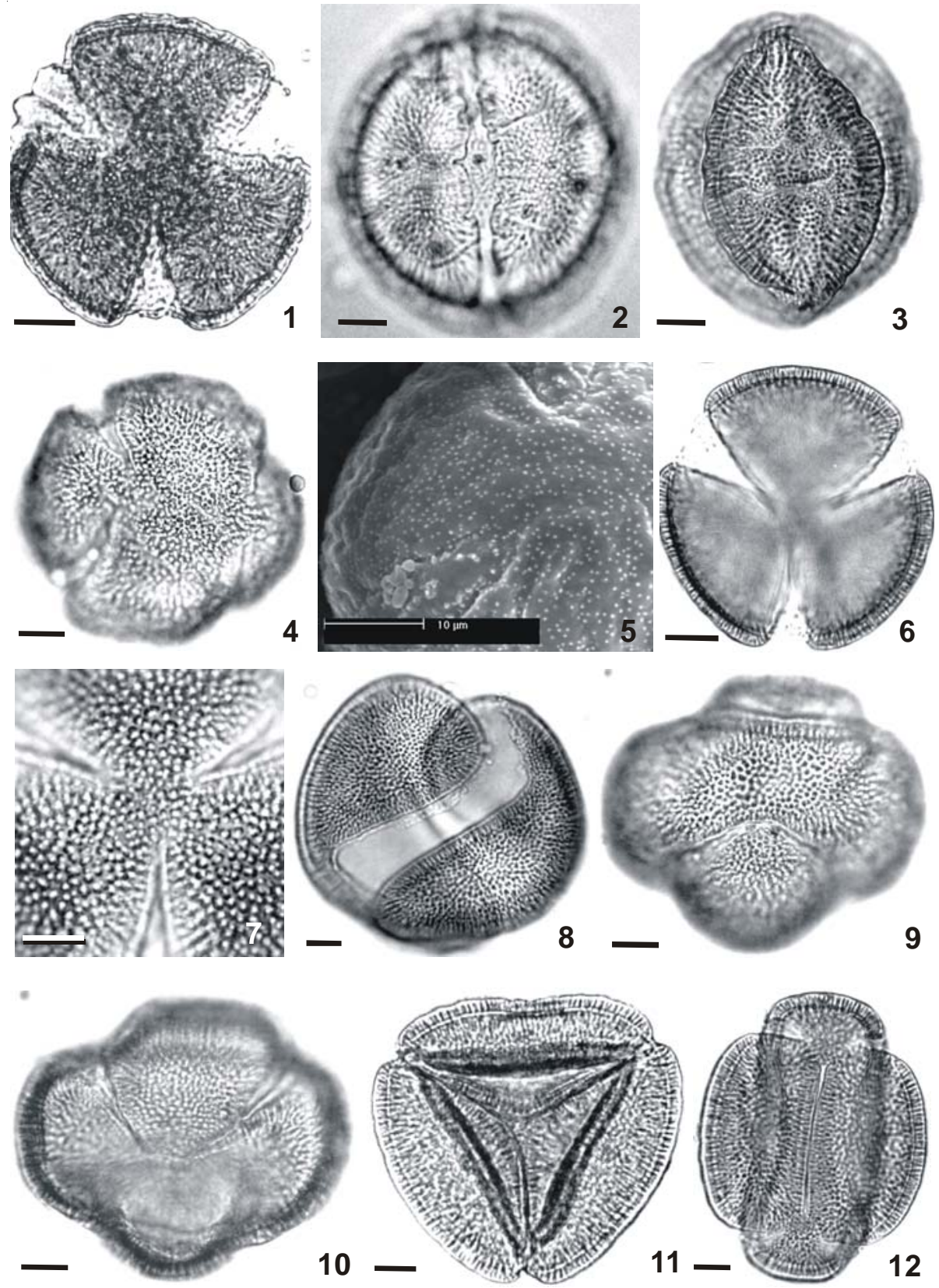

Figuras 1-3. Merremia aegyptia (L.) Urban: 1. Vista polar. 2. Vista equatorial, colpo. 3. Vista equatorial, superfície. Figuras 4-5. M. cissoides (Lam.) Hall.: 4. Vista Polar grão de pólen 4-colpado. 5. Superfície (MEV). Figuras 6-12. M. digitata (Sprengel) Hall. var. digitata: 6. Vista polar, corte óptico. 7. Detalhe do apocolpo; 8. Grão de pólen irregularmente colpado. 9-10. Grão de pólen 4(sin)colpado. 11-12. Grão de pólen 6-pantocolpado. (Escala microscopia óptica =10 $\mu \mathrm{m}$ ). 
foi observado por Sengupta (1972), Ferguson et al (1977) e Palácios-Chávez et al. (1991). O’Donell (1941) colocou M. umbellata como única espécie pertencente à Merremia sect. Xanthips (Griseb.) Hall, por apresentar flores agrupadas em dicásios umbeliformes; o tipo apertural dos grãos de pólen desta espécie corrobora sua posição taxonômica distinta ante as demais espécies estudadas.

Dentre as espécies pertencentes a Merremia sect. Cissoides (House) O'Donell, M. tomentosa
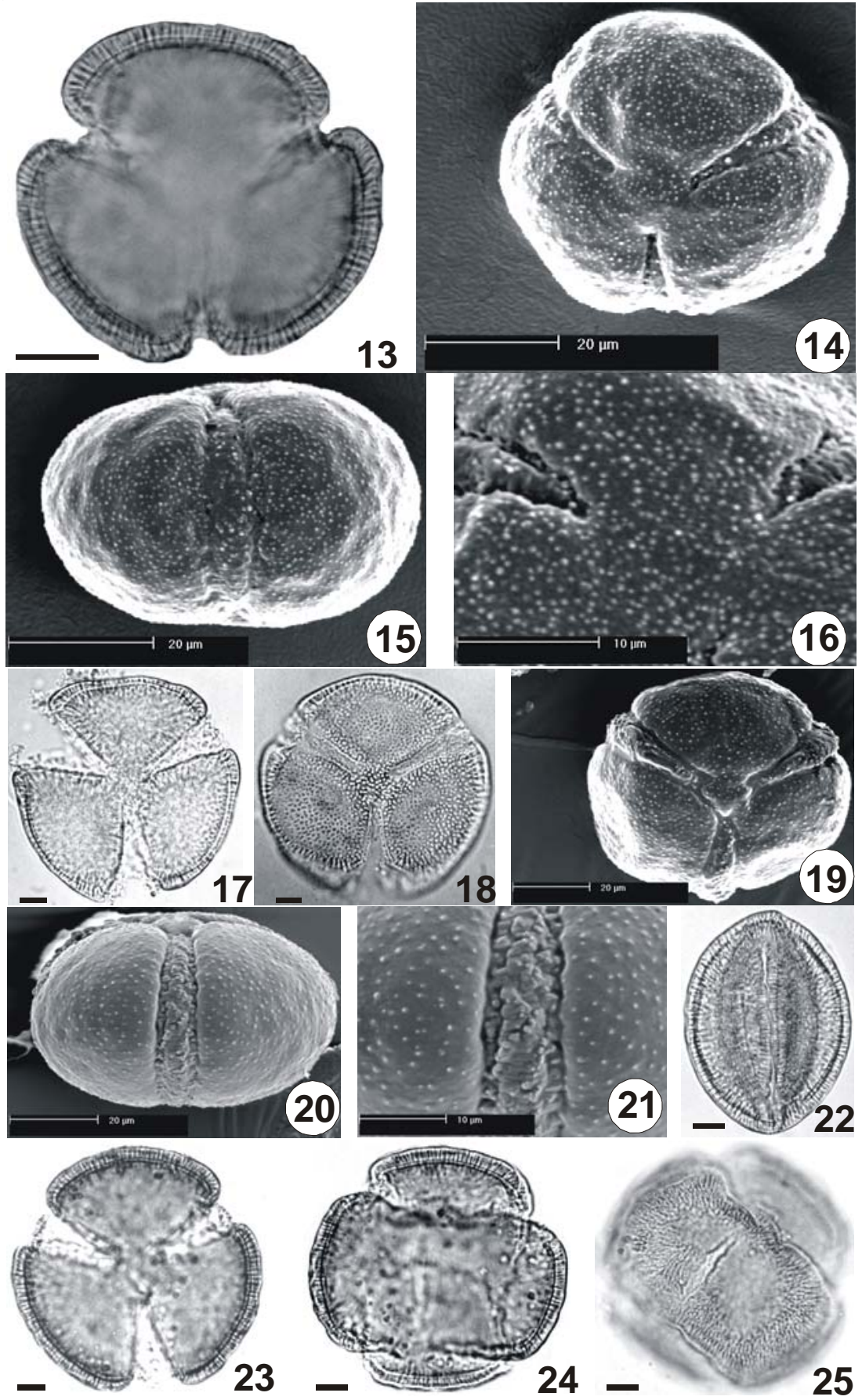

Figuras 13-16. Merremia digitata var. ericoides (Meisn.) Austin \& Staples: 13-14. Vista polar, corte óptico e superfície (MEV), respectivamente. 15. Superfície. 16. Detalhe do apocolpo (MEV). Figuras 17-25. M. dissecta (Jacq.) Hall. var. edentata: 17-19. Vista polar, corte óptico e superfície (MO e MEV), respectivamente. 20. Vista equatorial (MEV). 21. Detalhe do colpo (MEV). 22-25. M. flagellaris (Choisy) O’Donell: 22. Vista equatorial (grão de pólen amassado). 23. Corte óptico, vista polar. 24-25. Grão de pólen 6-pantocolpado, corte óptico e superfície, respectivamente. (Escala microscopia óptica =10 $\mu \mathrm{m}$ ). 
apresentou, assim como M. aegyptia, M. dissecta var. edentata e $M$. macrocalyx apenas grãos de pólen 3-colpados nos espécimes analisados.

Outros tipos polínicos foram observados em espécies de Merremia, conforme a literatura analisada. Ferguson et al. (1977), estudando Merremia e Operculina, observaram grãos de pólen 5-zonocolpados (em M. vitifolia Hall. f.), 9-12 pantocolpados
(M. siribica Hall. f.), 12-pantocolpados (M. pinnata Hall. f.) e até pantoporados (M. tridentata Hall. f. e M. medium Hall. f.).

Muitos orbículos foram observados nas lâminas preparadas dos espécimes de $M$. aegyptia e M. macrocalyx, não sendo observados nas demais espécies. Muitas vezes, os orbículos têm relevância taxonômica, pois apresentam uma morfologia bastante
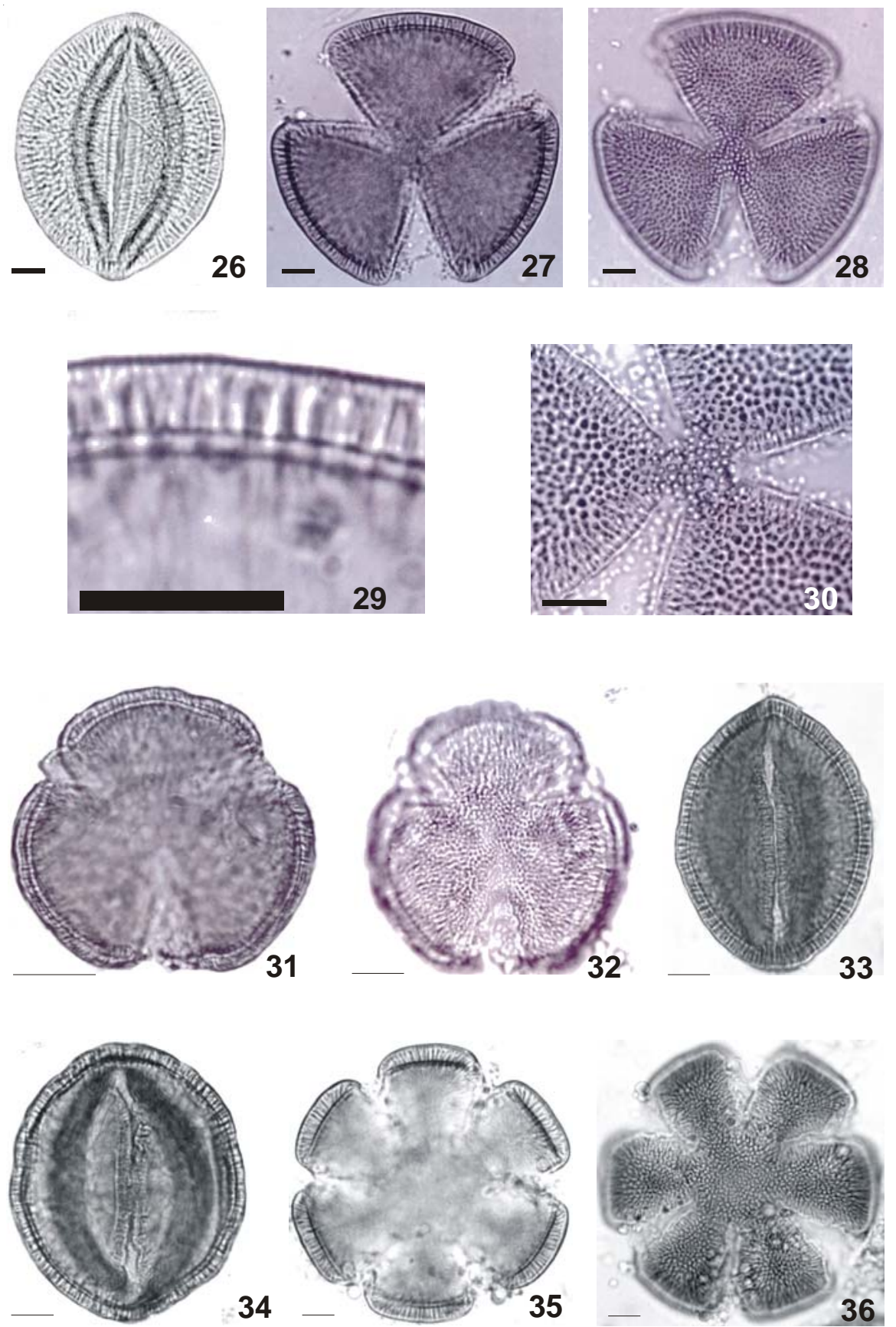

Figuras 26-30. Merremia macrocalyx (Ruiz et Pav.) O’Donell: 26. Vista equatorial. 27-28. Vista polar, corte óptico e superfície, respectivamente. 29. Detalhe da exina em corte óptico. 30. Detalhe do apocolpo. 31-33. M. tomentosa (Choisy) Hall.: 31. Vista polar, corte óptico. 32. Vista polar, superfície. 33. Colpo de contorno irregular. 34-36. M. umbellata (L.) Hall.: 34. Vista equatorial, corte óptico. 35. Vista polar, corte óptico. 36. Vista polar, superfície. $($ Escala $=10 \mu \mathrm{m})$. 
variada mas não sendo possível ser visualizada apenas sob microscopia óptica.

Os dados obtidos revelam que as seções $M$. sect. Schizips e M. sect. Xanthips propostas por O'Donell (1941) para as espécies de Merremia estão confirmadas com base também nas características palinológicas; $M$. sect. Cissoides forma um grupo estenopolínico do qual diverge $M$. tomentosa por apresentar apenas grãos de pólen com três aberturas.

\section{Referências bibliográficas}

Austin, D.F. 1979. Studies of the Florida Convolvulaceae II. Merremia. Florida Scientist 42(4): 216-222.

Austin, D.F. \& Staples G.W. 1983. Additions and changes in the neotropical Convolvulaceae - notes on Merremia, Operculina, and Turbina. Journal Arnold Arboretum 64: 483-489.

Araújo, R.C.M.S.; Leite, K.R.B. \& Santos, F.A.R. 2000. Morfologia polínica de Convolvulaceae da Bahia - Brasil. Revista da Universidade de Guarulhos - Geociências 5: 208-211.

Barroso, G.M.; Peixoto, A.L.; Ichaso, C.L.F.; Costa, C.G.; Guimarães, E.F. \& Lima, H.C. 1986. Sistemática de Angiospermas do Brasil. v.3. Viçosa, Universidade Federal de Viçosa.

Carreira, L.M.M. \& Barth, O.M. 2003. Atlas de pólen da vegetação de canga da Serra de Carajás. Belém, Museu Paraense Emílio Goeldi.

Dajoz, I.; Mignot, A.; Hoss, C. \& Tile-Bottraud, I. 1995. Pollen aperture heteromorphism is not due to unreduced gametophytes. American Journal Botany 82(1): 104-111.

Erdtman, G. 1952. Pollen morphology and plant taxonomyAngiosperms. Stockholm, Almqvist \& Wiksell.

Erdtman, G. 1960. The acetolysis method. A revised description. Svensk Botanisk Tidskrift 39: 561-564.

Ferguson, I.K.; Verdcourt, B. \& Poole, M.M. 1977. Pollen morphology in the genera Merremia and Operculina (Convolvulaceae) and its taxonomic significance. Kew Bulletin 31(4): 763-773.

Hallier, H.J.G. 1893. Versuch einer naturlichen gliederung der Convolvulaceae. Botanical Journal Arboretum 16: 479-591.
Holmgren, P.K.; Holmgren, N.H. \& Barnett, L. 1990. Index Herbariorum. New York, New York Botanical Garden.

Laguardia, A.M. 1961. Morfologia del grano de pollen de algumas Convolvulaceas uruguayas. Boletim Sociedad Argentina Botánica 9: 187-197.

Leuenberger, E. 1976. Die pollenmorphologie der Cactaceae. Dissertationes botanicae 31: 1-321.

Lewis, W.H. \& Oliver, R.L. 1965. Realignment of Calystegia and Convolvulus (Convolvulaceae). Annals of the Missouri Botanical Garden 52: 217-222.

Mabberley, D.J. 1987. The plant-book. Cambridge, Cambridge University Press.

Meissner, C.F. 1869. Convolvulaceae. Pp. 72-124, 199-370. In: C.P.F. Martius \& A.G. Eichler (eds.). Flora brasiliensis. Lipsiae, F. Flischer.

Melhem, T.S. \& Corrêa, A.M.S. 1987. Flora polínica da Reserva do Parque Estadual das Fontes do Ipiranga (São Paulo, Brasil) - Família 137-Convolvulaceae. Hoehnea 14: $15-23$.

O'Donell, C.A. 1941. Revision de las especies americanas de Merremia. Lilloa 6: 467-554.

Palacios-Chávez, R.; Ludlow-Wiechers, B. \& Villanueva, G. R. 1991. Flora palinológica de la Reserva de La Biosfera de Sian Ka'na, Quintana Roo, Mexico. Mexico, Centro de Investigaciones de Quintana Roo.

Pozhidaev, A.E. 2000. Pollen variety and aperture patterning. Pp. 205-225. In: M.M. Harley; C.M. Moton \& S. Blackmore (eds.). Pollen and Spores: Morphology and biology. London, Royal Botanic Gardens, Kew, Richmond.

Punt, W.; Blackmore, S.; Nilsson, S. \& Le Thomas, A. 1994. Glossary of pollen and spores terminology. $2^{\text {nd }} e d$. Utrecht, LPP Foundation.

Ribeiro, J.E.L.S. \& Bianchini, R.S. 1999. Convolvulaceae. Pp. 588-591. In: J.E.L.S. Ribeiro; M.J.G. Hopkins; A. Vicentini; C.A.S. Sothers; M.A.S. Costa; J.M. Brito; M.A.D. Souza; L.H.P. Martins; L.G. Lohman; P.A.C.L. Assunção; E.C. Pereira; C.F. Silva; M.R. Mesquita \& L.C. Procópio. (eds.). Flora da Reserva Duck: Guia de identificação das plantas vasculares de uma floresta de terra-firme na Amazônia Central. Utrecht, INPA.

Sengupta, S. 1972. On the pollen morphology of Convolvulaceae with special reference to taxonomy. Review of Paleobotany and Palynology 13: 157-212.

Wodehouse, R.P. 1935. Pollen grains. Their structure, identification and significance. New York, McGraw-Hill Book Company. 